SUPPORTING INFORMATION

\title{
Polymer Discs with High Interfacial Adhesion Fabricated from Hot-Pressing of Microspheres
}

Hong Zhang, ${ }^{\mathrm{a}, \mathrm{b} \dagger}$ Waranyou Tuntanatewin, ${ }^{\mathrm{c} \dagger}$ Kenichi Ishikura, ${ }^{\mathrm{d}}$ Daiki Sogabe, ${ }^{\mathrm{d}}$ Kodai Sugawara, ${ }^{\mathrm{a}}$ Akira Tokui, ${ }^{\mathrm{d}}$ Atsushi Nakagawa, ${ }^{\mathrm{b}}$ and Yosuke Okamura ${ }^{\mathrm{a}, \mathrm{b}, \mathrm{c}, \mathrm{d}^{*}}$

aDepartment of Applied Chemistry, School of Engineering, Tokai University, 4-1-1 Kitakaname, Hiratsuka, Kanagawa 259-1292, Japan

bMicro/Nano Technology Center, Tokai University, 4-1-1 Kitakaname, Hiratsuka, Kanagawa 2591292, Japan

${ }^{\mathrm{c}}$ Course of Science and Technology, Graduate School of Science and Technology, Tokai University, 4-1-1 Kitakaname, Hiratsuka, Kanagawa 259-1292, Japan

${ }^{\mathrm{d}}$ Course of Applied Science, Graduate School of Engineering, Tokai University, 4-1-1 Kitakaname, Hiratsuka, Kanagawa 259-1292, Japan

${ }^{\dagger}$ H.Z. and W.T. contributed equally to this work.

*E-mail: y.okamura@tokai-u.jp. 

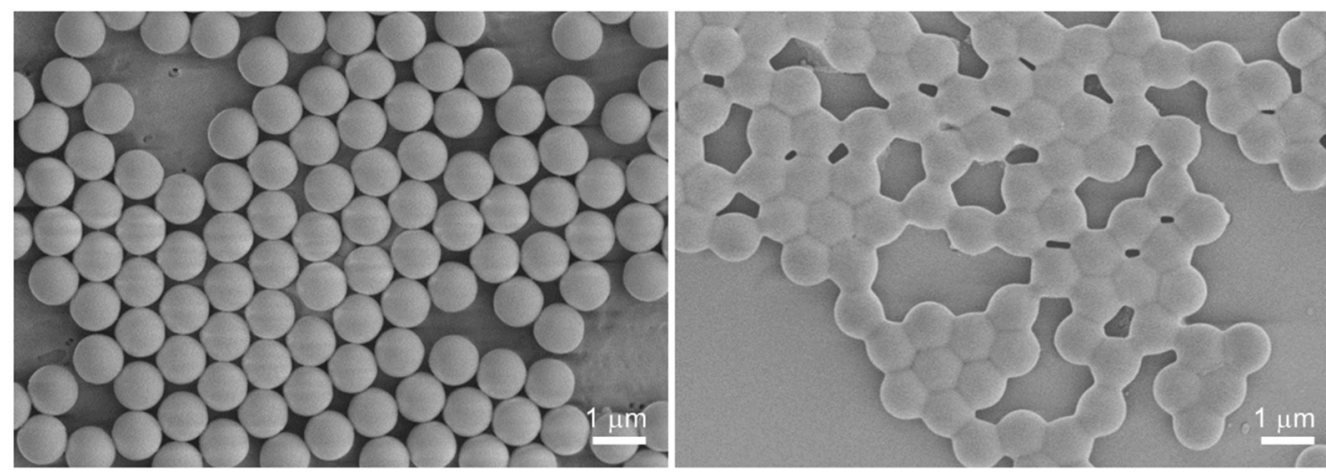

Figure S1. SEM images for PS microspheres (ca. $1.0 \mu \mathrm{m}$ in diameter) without sacrificial matrix (A) before and (B) after heated at $110^{\circ} \mathrm{C}$ on a hot plate.
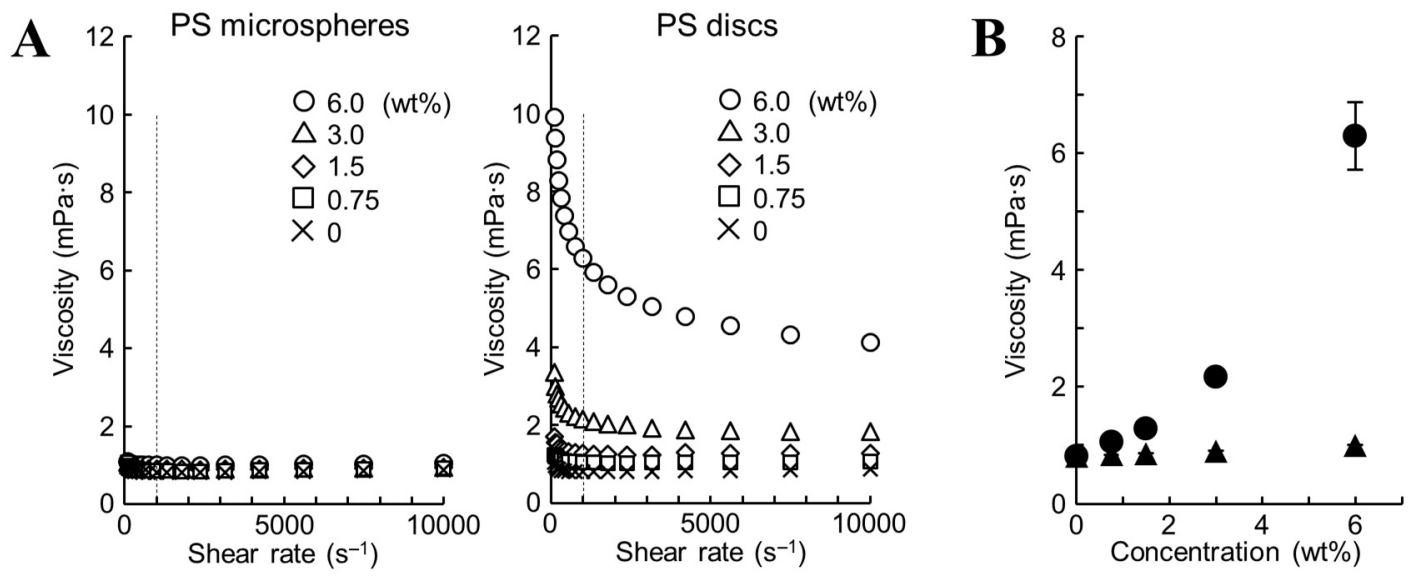

Figure S2. (A) Viscosity of PS microspheres and PS discs (ca. $1.0 \mu \mathrm{m}$ in diameter) suspension at various particle concentrations and shear rates. (B) Viscosity at the shear rate of $237 \mathrm{~s}^{-1}$ from both microspheres (triangle) and discs (circle) versus the particle concentration in suspension $(N=3-$ 4 , data given as mean $\pm \mathrm{SD})$. 
$\mathbf{A}$

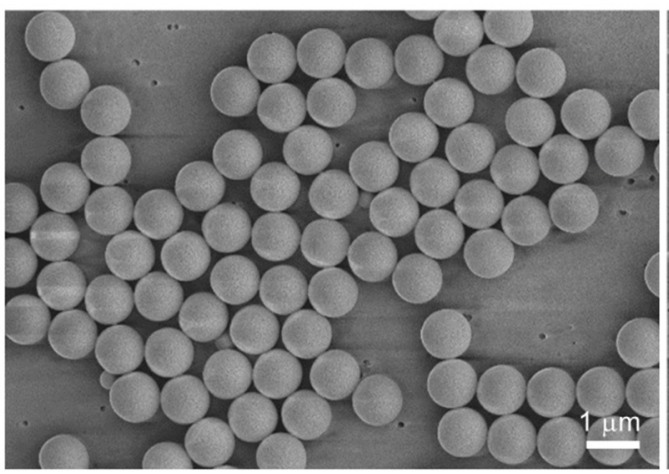

B

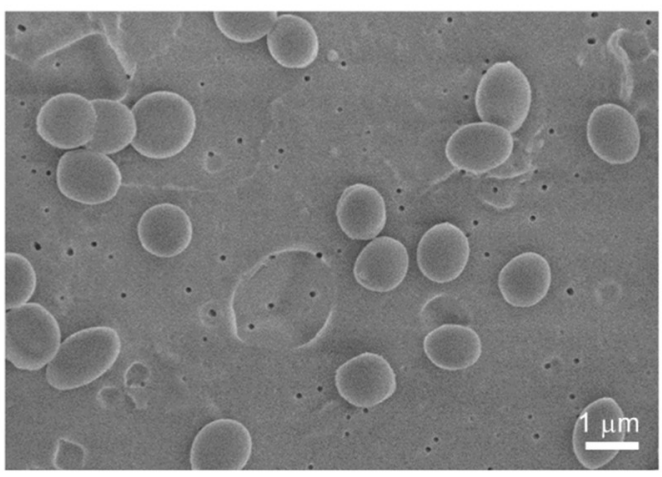

Figure S3. SEM images for PS microspheres (ca. $1.0 \mu \mathrm{m}$ in diameter) embedded in PVA sacrificial film instead of alginate gel (A) before and (B) after hot-press. 

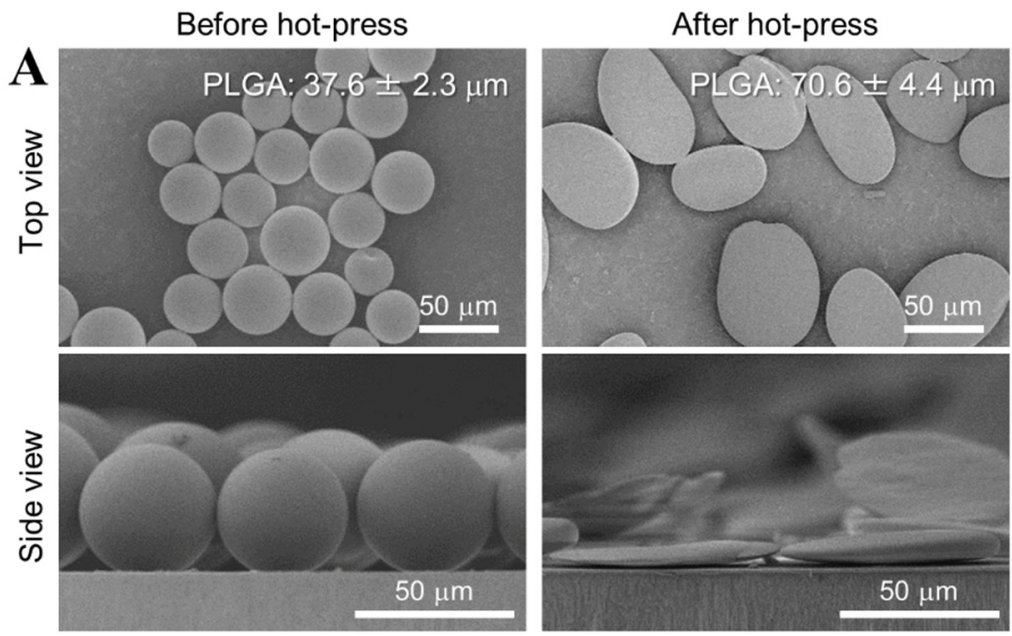

B

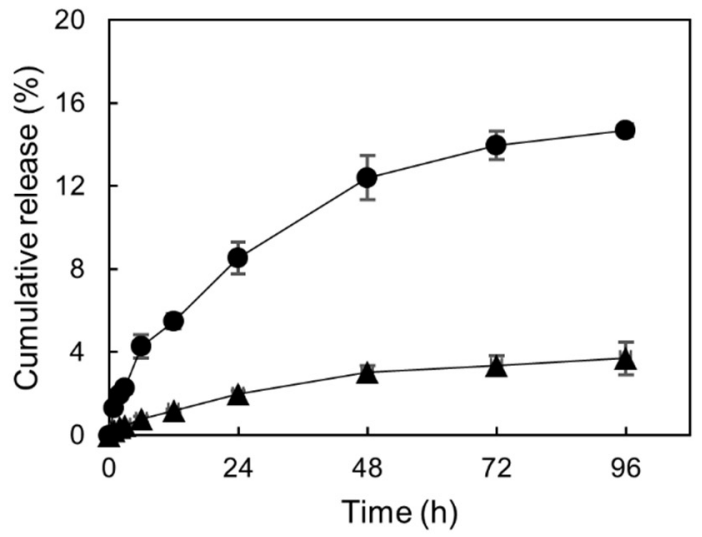

Figure S4. Loading and release behaviors of PLGA particles. (A) SEM images (top view and cross-sectional view) for the fabricated PLGA microspheres before and after hot-press. (B) Cumulative release profile of $\mathrm{DiOC}_{2}(3)$ from PLGA microspheres (triangle) and PLGA discs (circle) suspended in $\mathrm{PBS}(\mathrm{pH}: 7.4)$ at $37^{\circ} \mathrm{C}(N=3$, data given as mean $\pm \mathrm{SD})$.

Movie S1. Aggregation assay by mixing and stirring BSA-coated PS microspheres and discs suspension with GA aqueous solution, played in real time. 